ACCEPTED MANUSCRIPT

\title{
Optical design of InGaN/GaN nanoLED arrays on a chip: toward: highly resolved illumination
}

To cite this article before publication: Katarzyna Kluczyk-Korch et al 2020 Nanotechnology in press https://doi.org/10.1088/1361-6528/abcd60

\section{Manuscript version: Accepted Manuscript}

Accepted Manuscript is "the version of the article accepted for publication including all changes made as a result of the peer review process, and which may also include the addition to the article by IOP Publishing of a header, an article ID, a cover sheet and/or an 'Accepted

Manuscript' watermark, but excluding any other editing, typesetting or other changes made by IOP Publishing and/or its licensors"

This Accepted Manuscript is @ 2020 IOP Publishing Ltd.

During the embargo period (the 12 month period from the publication of the Version of Record of this article), the Accepted Manuscript is fully protected by copyright and cannot be reused or reposted elsewhere.

As the Version of Record of this article is going to be / has been published on a subscription basis, this Accepted Manuscript is available for reuse under a CC BY-NC-ND 3.0 licence after the 12 month embargo period.

After the embargo period, everyone is permitted to use copy and redistribute this article for non-commercial purposes only, provided that they adhere to all the terms of the licence https://creativecommons.org/licences/by-nc-nd/3.0

Although reasonable endeavours have been taken to obtain all necessary permissions from third parties to include their copyrighted content within this article, their full citation and copyright line may not be present in this Accepted Manuscript version. Before using any content from this article, please refer to the Version of Record on IOPscience once published for full citation and copyright details, as permissions will likely be required. All third party content is fully copyright protected, unless specifically stated otherwise in the figure caption in the Version of Record.

View the article online for updates and enhancements. 


\section{Optical design of InGaN/GaN nanoLED arrays on a chip: toward highly resolved illumination}

K Kluczyk-Korch ${ }^{1}$, D Palazzo ${ }^{1}$, A Waag $^{2,3}$, Á Diéguez ${ }^{4}$, J D Prades $^{4}$, A Di Carlo ${ }^{1,5}$ and M Auf der Maur ${ }^{1}$

${ }^{1}$ Department of Electronic Engineering, University of Rome "Tor Vergata", Via del Politechnico 1, 00133 Rome, Italy, e-mail: katarzyna.kluczyk@uniroma2.it

${ }^{2}$ Institute for Semiconductor Technology, University of Technology Braunschweig, Braunschweig, Germany

${ }^{3}$ Laboratory for Emerging Nanometrology LENA, Braunschweig, Germany

${ }^{4}$ Department of Electronic and Biomedical Engineering, Universitat de Barcelona, Barcelona, Spain ${ }^{5}$ ISM-CNR, Via Fosso del Cavaliere 100, 00133 Rome, Italy

E-mail: katarzyna.kluczyk@uniroma2.it

August 2020

Abstract. The physical laws of diffraction limit the spatial resolution of optical systems. In contrary to most superresolution microscopy approaches used today, in our novel idea we are aiming to overcome this limit by developing a spatially resolved illumination source based on semiconductor nanoscale Light Emitting Diode (nanoLED) arrays with individual pixel control. We present and discuss the results of optical simulations performed for such nanoLED emitter arrays and analyze the theoretical limits of this approach. As possible designs we study arrays of GaN nanofins and nanorods (obtained by etching nanofin arrays), with InGaN/GaN multi quantum wells embedded as active regions. We find that a suitable choice of the array dimensions leads to a reasonably directed light output and concentration of the optical power in the near field around an activated pixel. As a consequence, the spatial resolution for this type of microscopy should only be limited by the pixel pitch, and no longer by the optical diffraction. Realization of optimized nanoLED arrays has a potential to open new field of chip based superresolution microscopy, making super-high spatial resolution ubiquitously available.

Keywords: LED arrays, superresolution microscopy, LED

\section{Introduction}

The physical laws of diffraction, as one of the consequences of the Heisenberg uncertainty principle, generally limit the spatial resolution of optical systems formulated by Abbe and Rayleigh to about $200 \mathrm{~nm}$ for light in the visible range $[1,2,3]$. This is a theoretical limit, which is already very hard to achieve. Most of the high-end optical microscopes 
today have a spatial resolution in the range of $400 \mathrm{~nm}$. The loss of spatial information is associated with a loss of evanescent waves upon propagation from a source to the detector. However, the near field interaction between the source and a sample allows to encode the spatial information carried by the evanescent waves and retrieve it in the far field, which is therefore a way to achieve super-resolution. There is a number of superresolution methods using both far field and near field techniques. In the first group one can find e.g. confocal microscopy, exploiting nonlinear effects (e.g. multiphoton excitation microscopy) or saturation effects (e.g. stimulated emission depletion (STED)) or structured illumination microscopy (SIM). In the second e.g. scanning tunneling optical microscope (STOM), which aim to directly collect the information contained in evanescent waves [1].

Some of the methods, e.g. photo-activated localization microscopy (PALM), stochastic optical reconstruction microscopy (STORM) or SIM, are based on image reconstruction rather than on direct measurements. In PALM and STORM techniques, it is necessary to gain and combine thousands of images for different positions of activated fluorophores [2]. In SIM several images for various angles between the structured light pattern and specimen [4] have to be captured. This causes a decrease of temporal resolution and makes it difficult to obtain super-resolved images of live cells or quantify their dynamics [5]. On the other hand, the STED technique has reasonably fast image integration time, but it requires high laser power excitation, which can damage the sample [3] and hence limits its applicability. Another drawback of most of these techniques, i.e. STED, PALM, STROM, SIM, is their requirement of fluorescent dyes or immunolabeling by antibodies specific to cellular antigens $[1,2,3]$.

Fluorescence label free super-resolution techniques, e.g. utilizing solid and liquid immersion lenses (i.e. micrometer dielectric spheres or half spheres) [6], optical oscillation [7], or metamaterials are also reported [1, 2]. However, usually they only play a supporting role to existing microscopy techniques, providing an additional component to the microscope.

Finally, all of these methods lead to bulky and large microscopes, and in some cases need specially prepared samples, highly skilled users, or expensive maintenance.

We recently proposed a different and novel approach to overcome the diffraction limit based on a spatially resolved light source using semiconductor nanoscale Light Emitting Diode (nanoLED) arrays with individual pixel control [8]. In this paper we further analyze the abilities and limitations of this concept using full wave electromagnetic simulations and look toward optimization of the system. We focus on LEDs due to their high brightness and switching speed with low overall power consumption in comparison to technologies like liquid crystal display (LCD) or organic LED (OLED). GaN is a suitable material platform, since the relatively low surface recombination velocity of electrons and holes allow to fabricate very small LEDs, in the $n m$ range, by still keeping up reasonably high efficiencies. The working principle is simple. The locally illuminated sample absorbs a certain portion of the light, which is then detected as a decrease in the intensity. Therefore, this approach does not require 
using dyes or immunolabeling. The spatial information is delivered by the point of illumination, and not by a complex lens system or the detector. A simple integrating photodiode can also be used as a detector. Without any requirement for a lens system, this technique has a potential to be integrated into small, portable, chip based optical devices capable of real-time imaging. More detailed description of this approach called Nano Illumination Microscopy (NIM) together with the results obtained from prototype microscope can be found in [8].

The key issue in this approach to super-resolution imaging is the development of the light engine, which requires a dense array of nano-sized light sources. For this reason, the present work focuses on the design strategies for GaN nanoLED arrays, as it has been found experimentally that nitride nanorod LEDs emit light down to diameters of roughly $100 \mathrm{~nm}[9,10,11,12]$. As for now, working $\mu L E D-b a s e d$ displays with pixel sizes down to $3.6 \mu \mathrm{m}$ and array dimensions of roughly $500 \times 500$ have been reported [13, 14, 15]. However, many of the results presented in the literature are settled in the region of 5 up to $100 \mu \mathrm{m}[16,13,14,17]$, and concentrate rather on superior driving and emission characteristics than on further miniaturization of the pixel size. Until now, microLED research is targeting micro-displays for augmented reality, and the single microLED is only switchable at video frame rates. This is far too slow for our application, where single nanoLEDs need to be switchable at least at $\mathrm{MHz}$ rate, independent from each other. The LEDs in the proposed system can indeed be switched at speed below $1 \mathrm{~ns}$. In particular, the array of $5 \mu \mathrm{m}$ sized LEDs has been driven with pulses of 690 ps. Up to date numerical simulations of optical properties of nanoLED arrays mostly focus on different applications, such as maximization for light extraction efficiency [18], e.g. photonic crystal LEDs [19] or nanowire solar cells [20]. Thus, near field electromagnetic field patterns, which are crucial for superresolution illumination, were not yet systematically studied. In this paper we analyze the electromagnetic near field, as well as the full width at half maximum (FWHM) of radiation patterns generated by a single pixel (both in an array and in a homogeneous environment) and finally show simulated images formed by the microscope. We aim to study and optimize the design of the LED array in terms of its optical properties, in particular with respect to the achievable resolution.

A nanoLED array should be designed in such a way as to maximize the light extraction from a particular LED, acting as a pixel, in the forward direction. We are interested in minimization of the spatial FWHM of the optical signal and maximizing its intensity. This can be achieved by guiding the emitted photons towards the relevant surface by a linear index-guided structure like a semiconductor nanorod embedded in a medium with lower refractive index. By restricting the nanorod width, it is possible to completely eliminate the transverse electric guiding modes, which results in enhancement of the light extraction efficiency and increment of directionality of the emission pattern [21]. On the other hand, light propagation in the plane perpendicular to the axis of the pixels can be controlled by periodicity and symmetry of the array.

The resolution of the final device depends on the FWHM of the signal extracted 
from a particular LED and on the value of the array period. One of the limitations is related to the sampling of the viewed object. To properly resolve an object with periodicity $P$, one should use sampling of at least twice smaller period $p<\frac{P}{2}$. Therefore, one should look for the smallest possible LED array periods. However, at the same time, the cross talk between pixels, resulting in complex spatial intensity distributions with multiple maxima and large FWHM, increases with decreasing distance between the LEDs.

\section{Methodology}

Maximization of the forward light extraction from a LED array requires an optimization of the light propagation efficiency towards the surface of the array. More precisely, it requires finding a geometry of the nanorod or nanofin array such that at the desired wavelength (here $450 \mathrm{~nm}$ as typical wavelength of blue emitting nitride LEDs) light propagation along the nanorods or nanofins is allowed while, ideally, the same frequency cannot propagate in the array plane.

In the case of large arrays, when the structure can be described as a photonic crystal, and the influence of boundaries can be neglected, the band diagram becomes a valuable topic of investigation for the array design.

An optimization of the structure can be then performed by finding a suitable periodicity and nanorod size to form a band gap and consequently quenching the waveguiding in the plane of the array. However, in smaller arrays, of a few tens of rods or fins, the boundary effects are expected to be significant and the periodic approach is not suitable. Therefore, we have performed full field simulations applying, in particular, the finite difference time domain (FDTD) method implemented in the commercial software CST Studio [22].

We have simulated the emission patterns from a single pixel (i.e. a single nanorod or nanofin) in nanorods and nanofins arrays. We have modeled arrays of $11 \mathrm{x} 11$ nanorods and 11 nanofins, using a single point dipole as a source of the radiation in the central pixel at a distance $\mathrm{H}_{2}$ from the top surface of the GaN (see Fig. 1). In most simulations we assumed that the distance between the dipole and the top surface of the pixel is equal to $400 \mathrm{~nm}$. This value is obtained by summing the typical distance between active region and GaN surface in InGaN LED technology with the thickness of passivation and dielectric layers. We have applied open boundary conditions in all directions in order to avoid reflections and interference at the model boundaries and periodic copies of the dipole emitter appearing in the case of periodic boundary conditions. The dielectric constants used in simulations are reported in table 1. Although usage of a single dipole as a source may appear to be a rough approximation, our simulations for a 3x3 matrix of dipole sources placed on a patch of the size of the rod cross section gives only a very smalldifference form the result with a single dipole at the center of the patch (see the supporting material).

The proposed device structure presented in Fig. 1 is based on a top-down matrix 
Table 1. Relative permittivity $\epsilon=\epsilon^{\prime}+i \epsilon^{\prime \prime}$ at $450 \mathrm{~nm}$ used in the calculations taken from $[24,25,26,27]$. The materials for which the imaginary part $\epsilon^{\prime \prime}$ of the permittivity is not stated were simulated as non-dispersive materials.

addressing technology described elsewhere [23]. Actually, we are comparing here two possible designs of the array. A fin array (see panel A in Fig. 1), and a rod array (see panel B in Fig. 1), the latter obtained by etching the top portion of the GaN fins. After growing a planar InGaN/GaN LED wafer on a sapphire substrate a combination of dry and wet etching steps is applied in order to achieve a high aspect ratio array of InGaN/GaN fins. In the next step, the chips are planarized for the subsequent pcontact formation with thin metal or transparent conductive oxide (e.g. indium tin oxide, ITO) lines. Typical applications of Nano Illumination Microscopy microscopy relate to biological and micro/nanoscale systems. Therefore, we assume a microfluidic system on top of the LED array to bring the sample into the observation chamber and maintain proper cultivation conditions (liquid medium, nutrients, temperature, etc.). We modeled this upper interface as an SU-8 layer for the microfluidic enclosure, followed by a water reservoir.

\section{Discussion of the results and conclusions}

\subsection{Nanofin and nanorod arrays}

The width of fins in the currently available LED arrays have the size of several $\mu \mathrm{m}$. As we have shown in a previous publication [23], such a fin supports multiple guiding modes and as a result the near field emission pattern has a complex spatial characteristic, which can lead to difficulties in interpretation of the shadow image from the microscope. One can see that power is apparently emitted from positions associated to the neighboring pixels. From these observations we deduce that better control of the emission pattern and reduction of optical crosstalk between pixels would be desirable. A possible solution is shown in the following section. Note that we consider only the optical effects here, while we expect also some amount of electrical diffusion along the nanofin, which would add up to the optical crosstalk.

Therefore, in order to design light sources suitable for near field super-resolution microscopy one should look for single mode propagation along the pixel structure. In the case of a GaN $\left(n_{G a N}=2.41\right)$ LED embedded in air, a waveguide with square cross section or the fin geometry start to support a second mode TE01 for widths $W \geq 100$ nm. Thus, we have chosen the widths of the pixels to be in the range $W \in(50,100)$ nm. 
A) fin architecture

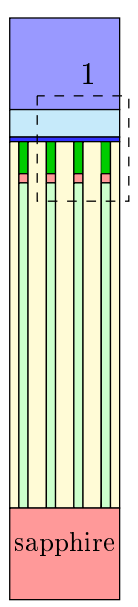

$\mathrm{xz}$ cross section

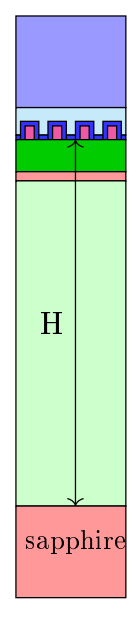

yz cross section

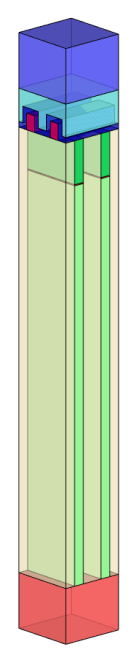
$\mathrm{y} \stackrel{\mathrm{z}}{ }$

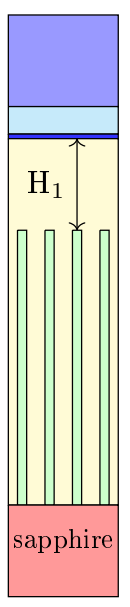

$\mathrm{xz}$ cross section

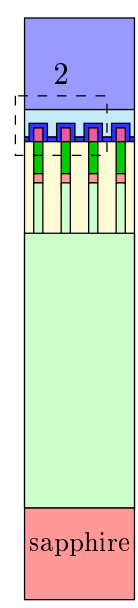

yz cross section

D) Voltage application scheme
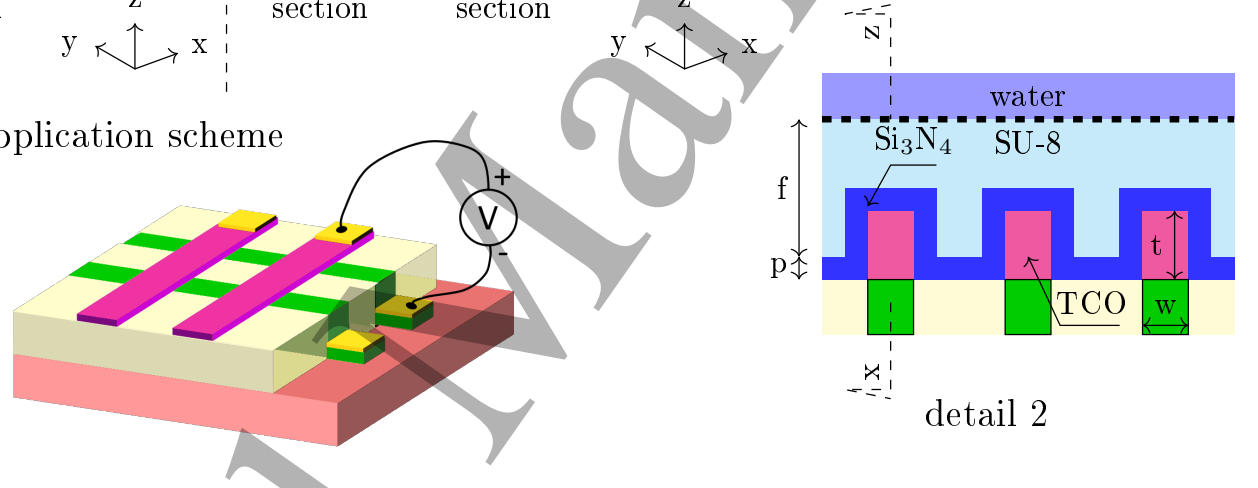

Figure 1. The scheme of the numerical model of fin (A) and rod (B) LED array based on the technology developed [23]. In the simulations we were changing width $w$ and period $P$ of the array. The other parameters were kept constant and equal to: $H=4$ $\mu \mathrm{m}, H_{1}=1 \mu \mathrm{m}, H_{2}=400 \mathrm{~nm}, p=50 \mathrm{~nm}, t=150 \mathrm{~nm}, f=300 \mathrm{~nm}, t=150 \mathrm{~nm}$. As a filler material, i.e. material located between the GaN domains we chose air, as we expect it to lead to the highest confinement of light inside GaN. Panel C shows the details of the top portion of the structure with respect to the regions indicated in A and $\mathrm{B}$ (dashed rectangles). Panel D shows schematically how the voltage is applied to the device.

Figure 2 shows the eyolution of the FWHM values of the signal generated by the central pixel of the LED array as a function of the array pitch. The values were calculated for three different widths of the LED fins (see subplot a) and rods (see subplot b). The distance $z$ at which FWHM of the signal was extracted was chosen to be the distance from the source to the object plane (SU-8/water interface), i. e. $700 \mathrm{~nm}$. Due to the crosstalk between the neighboring pixels for small array periods the resulting illumination signal exhibits multiple maximums. Therefore, the values presented in Fig. 2 correspond to the largest distance between the points for which the intensity of the signal was equal to the half of its global maximum, i.e. for the signals having multiple maximums we indicate the distance between the farthest external maximums. 
A planar InGaN/GaN MQW structure would emit equally with $x$ and $y$ (in-plane) polarization. In a structured device we expect a change in the relative contribution of different polarizations, therefore we report the FWHM in $x$ and $y$ direction for both dipole sources aligned along $x$ and $y$. The FWHM values depend on the array period and pixel width and vary between approximately $220 \mathrm{~nm}$ for large periods and $1100 \mathrm{~nm}$ for the small ones. The geometry of the device, i.e. periodic parallel layers of GaN and orthogonal ITO top contact crossbars, results in anisotropy of the device, which is visible also in the differences in the FWHM profiles. Nevertheless, due to increasing crosstalk between the neighboring pixels, for all the polarizations and directions of FWHM signal we observe a significant increase of FWHM value as the array period decreases. The influence of the dipole polarization is not strong in the case of rods, but it is observable for fins, which for $y$ polarized source shows stronger crosstalk compared to $x$ polarization, resulting in occurrence of multiple maximums for larger periods (up to $P=400 \mathrm{~nm}$ ).

Nanofins have larger FWHM in $y$ direction in comparison to nanorods for array periods $P$ lower than approximately $400 \mathrm{~nm}$. We consider this to be a result of free propagation of the electromagnetic field along the fin plane, which is orthogonal to the $x$ axis (see Fig. 1). In the case of small periods, the signal at the top of InGaN/GaN stack excites ITO neighboring bars with similar strength, causing additional maximums to appear. This is visible in the near field power plots shown in the Fig. 3. The additional maximums occur above crossings with ITO, which is also the position of the neighboring pixels. The slight elongation of the FWHM in $x$ direction, comparable in the case of fins and rods is caused by propagation of light along the ITO crossbars. One can also observe that the fins and the rods of smaller widths tend to have larger FWHM both for $x$ and $y$ directions. We relate this to a/smaller mode confinement factor for the lower widths of the waveguides and resulting slower spatial decay of the electromagnetic field in the medium surrounding the guide.

Figure 3 compares near field electromagnetic field power intensities for fin and rod geometry for different cross sections. The value of the array period and the pixel width are here the same and are equal to $P=300 \mathrm{~nm}$ and $W=100 \mathrm{~nm}$, respectively. Again, one can notice free propagation of the electromagnetic field along the fin in $y$ direction (top middle) and guiding of the field in the $x$, orthogonal direction. Green lines indicate the FWHM of the signal while the blue line show the positions of interfaces of each device.

Comparing the distribution of the electromagnetic field in the fin and the rod in $y z$ plane, we notice much larger FWHM at the top of GaN (middle blue line) in the case of the fin, which results in additional maximums of the signal at SU-8/water interface.

The etching of the top portion of the GaN fins at least partially optically decouples the neighboring pixels laying along the same fin. This hinders the electromagnetic field propagation in the fin plane and squeezes the FWHM in the fin direction. In particular, we do not observe the emission from the neighboring pixels, as it was the case for the fin arrays with periods smaller than $P<400 \mathrm{~nm}$ (see Fig.2). 
polarization x, FWHM x

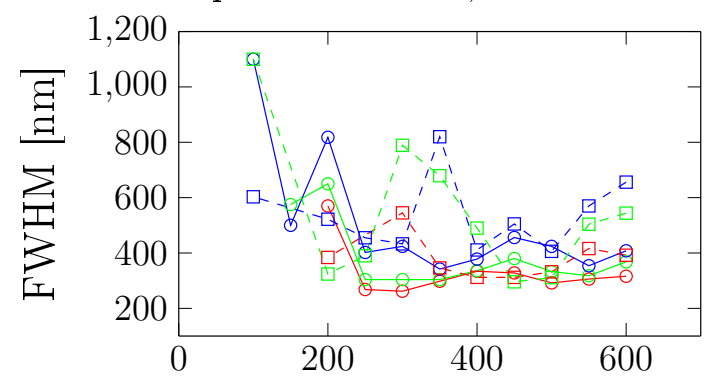

polarization y, FWHM x

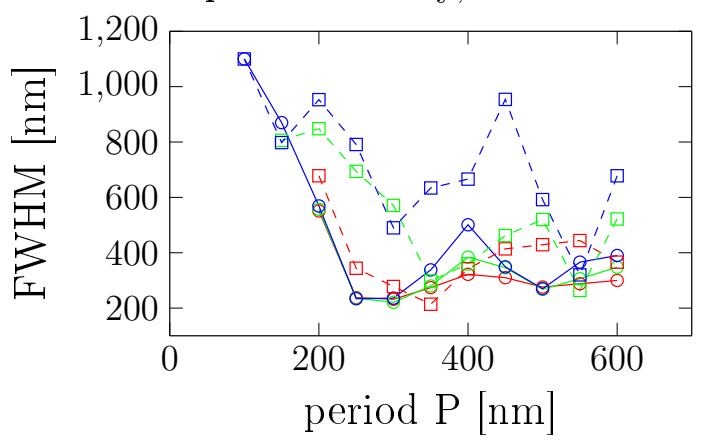

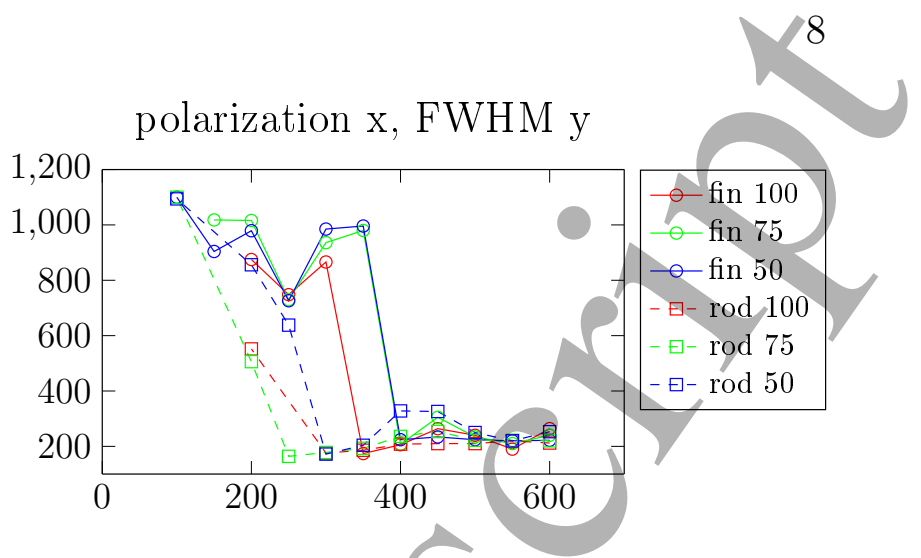

polarization y, FWHM y

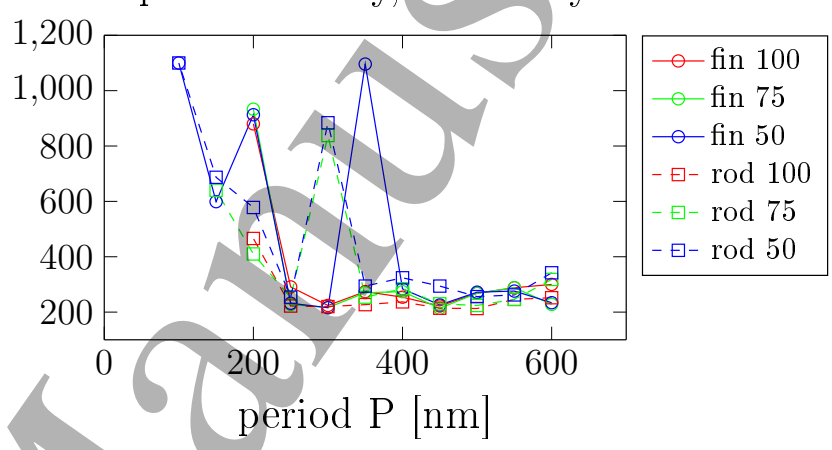

Figure 2. The FWHM of the light beam emitted from the central pixel taken at the object plane (SU-8/water interface, see Fig. 1) as a function of the array period $P$ and width $W$ of the rods/fins. The panels show FWHM along $x$ or $y$ direction for one of the two orthogonal polarizations.

\subsection{Single pixel characteristics? nanorod vs nanofin}

In order to better understand the ultimate limits for the possible concentration of the optical fields, we performed simulations on an isolated single rod and fin. The left side of Fig. 4 compares the FWHM of the extracted light signal as a function of distance from the source in the case of single rod and fin pixel geometries with varying widths $W$. The values were compared with the results for a dipole in a homogeneous environment and the position of the active region was fixed and equal to $H_{2}=400 \mathrm{~nm}$. As expected, the FWHM increases with the increase of the distance from the source, but due to a number of different layers in the LED stack complex behavior in the top LED region is observed. One can notice that the FWHM value for all of the rods and the fins is smaller than the one for a dipole in an homogeneous environment. The waveguide properties of the pixel structure seem to prevent spreading of the light and therefore effectively shift the location of the light source. The smallest FWHM is obtained for rods of $W=100$ $\mathrm{nm}$, which have the highest mode confinement factor. Furthermore the structures of small confinement factor (smaller $W$ ) have larger FWHM. The visible elongation of the signal cross section in $x$ direction is caused by the ITO crossbars.

On the right hand side of the Fig. 4, we show the comparison of FWHM for a single rod of width equal to $W=100 \mathrm{~nm}$ and an array of rods of the same width with different periodicities $P$. Due to the interaction between the pixels, the evolution of the signal 
FWHM for the arrays of LEDs is not a linear function of distance from the source. Moreover, its value increases with the decrease of the array period. Interestingly, for the period equal to $P=400 \mathrm{~nm}$ the values of FWHM are lower than the values for a single rod.

\subsection{Estimation of the resolution capabilities by imaging of metallic and dielectric} nanoparticles

As we explained in the introduction, the resolution of the NIM microscope depends both on the period of the illumination engine and on the characteristics of the optical signal, i.e. size and intensity of individual pixels. We have chosen two sets of geometric

Figure 3. Logarithm of the $z$ component of the Poynting vector $P_{z}$ emitted from a dipole source located at a distance $H_{1}=400 \mathrm{~nm}$ below the top surface of the GaN layer. Power distribution is shown for rod array (A) and fin array (B). The width $W$ of the fins and rods is equal to $100 \mathrm{~nm}$ and the period $P$ of the arrays is equal to $300 \mathrm{~nm}$. The green lines indicate the FWHM of the signal, while the blue lines indicate, starting from left, the positions of interfaces between sapphire and GaN, GaN and ITO, SU-8 and water. The last one is also the object plane. In $\mathrm{C}$ we show the magnifications of the field distributions near the top surface of the nanoLED array. 
parameters, including width $W$, period $P$ and dipole source distance from the top surface of the LED $\mathrm{H}_{2}$, and simulated the total intensity of outgoing light from each of the pixels while illuminating. At first we have calculated the pattern for two metallic Au spheres, as they have a large extinction cross section, which should ensure a significant signal contrast. Subsequently, finding well resolved images for Au spheres, we have repeated the simulations for the case of dielectric spheres. To model the latter we have used the dielectric function corresponding to liver tissue after [28]. According to the sampling theory, the resolution of a device is bounded by sampling frequency and one can resolve objects twice as large as the sampling distance.

In the proposed microscope design, a single CMOS Single Photon Avalanche Photodiode (SPAD) is employed as a detector. It collects and integrates the light coming out of each pixel in a certain range of angles, so that the system resolution is determined by the light spot size and pitch of the emitter array. Therefore, smaller size of the pixels and the array period should lead to better performance in terms of 
the resolution. However, the signal FWHM is also important, and it is affected by the array and pixel geometric structure, as we discussed in the previous sections 3.1, 3.2. Taking this into account, we decided to simulate the intensity maps from a dense array of small LEDs $\left(W=50 \mathrm{~nm}, P=100 \mathrm{~nm}\right.$ and $\left.H_{2}=150 \mathrm{~nm}\right)$ and a more sparse array ( $W=100 \mathrm{~nm}, P=300 \mathrm{~nm}$ and $H_{2}=400 \mathrm{~nm}$ ). The latter is the densest one, for which the crosstalk between the pixels is not observed. In both cases we simulated rod LED arrays, because of their narrower signal cross section in comparison with the fins for which we observe a large elongation of the signal FWHM in $y$-direction for $x$-polarized source.

The maps, together with the corresponding light intensity patterns impinging on the object plane, are shown in Fig.5. Gold spheres of radius equal to $50 \mathrm{~nm}$ were placed centrally above the two pixels in the center at a distance equal to the double LED array period (see scheme at Fig.5). The obtained images clearly show the difference in performance of these two microscopes, which from the point of/view of sampling theory and geometric optics should be similar. In case of the dense microscope (on the left in Fig. 5) two spheres cannot be clearly resolved, while for the sparse microscope (on the right in Fig. 5) we can observe separate dark spots due to the presence of the gold spheres. This can be explained by looking at the extracted power intensity spectra at the image plane, i.e. impinging on the spheres, in these two cases, shown in the bottom panels of the Fig.5. The signal from the dense LED array is two orders of magnitude less intense and significantly broader than the signal from the sparse array. In particular, the width of the FWHM signal of the dense array is significantly larger than the distance between the spheres. On the other hand, for the sparse array the FWHM width is smaller than the distance between the spheres and the resolution limit in this case mainly results from the LED array period. In the case of dielectric spheres (see plot (E) at Fig.5) the spheres are still visible, however the contrast of the image is worse. The spots corresponding to the spheres are now brighter than the background, due to the increased forward scattering of the spheres and small absorption coefficient of the dielectric material.

\section{Conclusions}

We have performed optimization studies for InGaN/GaN nanoLED arrays with possible application as NIM microscope light engine. We analyzed two pixels geometries: a 2D fin array and arrays of 3D patterned nanorods resulting from etching the $2 \mathrm{D}$ fin structure. The best performance was found for a nanorod array of pitch equal to $P=300 \mathrm{~nm}$, with a resolution equal to approximately $600 \mathrm{~nm}$.

One of the critical parameters for the NIM microscope engine is the FWHM of the light signal extracted from an individual pixel, which is going to illuminate the sample. This parameter depends on the waveguiding properties of the array, in particular on the crosstalk between the neighboring pixels. The crosstalk can be reduced by etching the fin LEDs and by proper choice of the resulting rods' width that maximizes the 


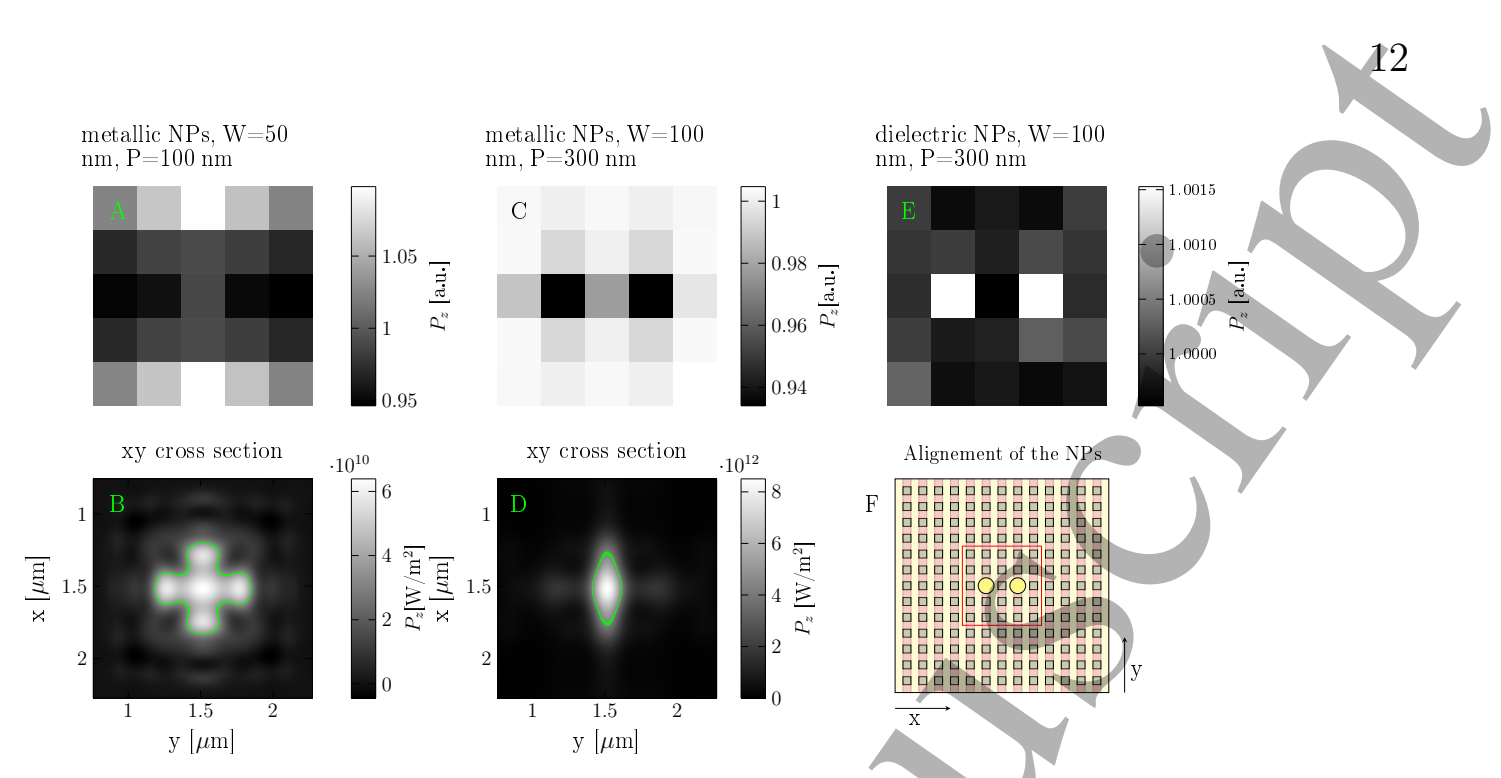

Figure 5. The maps of outgoing light power (z-component of the Poynting vector) calculated for each pixel in the array of LEDs for system of $(\mathrm{A}, \mathrm{C})$ two Au spheres or (E) two dielectric spheres. (A) The intensity map from a rod array of width $W=50$ $\mathrm{nm}$ and period $P=100 \mathrm{~nm}$ for two Au spheres, (C) from rod array of width $W=100$ $\mathrm{nm}$ and $P=300 \mathrm{~nm}$ for two Au spheres, (E) from rod array of width $W=100 \mathrm{~nm}$ and $P=300 \mathrm{~nm}$ and dielectric spheres. Panels (B and D) show the electromagnetic field distribution in the image plane (SU-8/water interface) generated by the central pixel corresponding to the maps (A and $\mathrm{C}$ ), respectively. The unpolarized dipole source was placed at distance $H_{2}=150 \mathrm{~nm}$ for the dense matrix (A) and $H_{2}=400 \mathrm{~nm}$ for the sparse matrix (C). Z-component of the Poynting vector was integrated over the top surface of the model. The panels ( $\mathrm{E}$ and $\mathrm{F}$ ) show the scheme of the model. In panel (E) we show only the central part of the array corresponding to the maps (A and C), i.e. the 25 central pixels of the 13 x 13 array.

guided mode confinement factor inside a pixel. Besides the problem of beam spread inside InGaN/GaN layers, the ITO crossbars can cause significant broadening of the light beam.

Further reduction of the grosstalk between the pixels may be achieved by improving the electromagnetic field confinement of the guided mode inside the pixel structure or, alternatively, by increasing the light absorption in between the pixels and preventing leakage of the electromagnetic field into the neighboring pixels. While the second approach leads to a significant drop in the intensity of the extracted light, the first one can possibly increase it, especially if used for thin waveguides, e.g. $W=50 \mathrm{~nm}$. Extremely skinned waveguides (e-skid), in which anisotropic cladding of the guide was shown to reduce the skin depth and strongly increase the mode confinement [29], seem to be a promising solution.

\section{Acknowledgement}

This work is being carried out in the European project Chipscope, funded by the European Union's Horizon 2020 research and innovation program under grant agreement no. 737089. J.D.P. acknowledges the support of the ICREA Academia 
Program and the DFG Project GrK NanoMet. A.W. acknowledges the support of Deutsche Forschungsgemeinschaft (DFG, German Research Foundation) under Germany's Excellence Strategy - EXC-2123 Quantum Frountiers - 390837967.

\section{References}

[1] L. Novotny and B. Hecht. Principles of Nano-Optics. Cambridge University Press, second edition, 2012.

[2] S. So, M. Kim, D. Lee, D. M. Nguyen, and J. Rho. Overcoming diffraction limit: From microscopy to nanoscopy. Applied Spectroscopy Reviews, 53(2-4):290 - 312, 2018.

[3] E. Sezgin. Super-resolution optical microscopy for studying membrane structure and dynamics. Journal of Physics: Condensed Matter, 29(27):273001, 2017.

[4] L. Schermelleh, Carlton P. M., S. Haase, L. Shao, L. Winoto, P. Kner, B. Burke, M. C. Cardoso, D. A. Agard, M. G. L. Gustafsson, H. Leonhardt, and J. W. Sedat. Subdiffraction Multicolor Imaging of the Nuclear Periphery with 3D Structured Illumination Microscopy. Science, 320(5881), 2008.

[5] R. Chéreau, J. Tønnesen, and U. V. Nägerl. STED mícroscopy for nanoscale imaging in living brain slices. Methods, 88(88):57 - 66, 2015.

[6] A. V. Maslov and V. N. Astratov. Resolution and Reciprocity in Microspherical Nanoscopy: Point-Spread Function Versus Photonic Nanojets. Physical Review Applied, 11(6):064004, 2019.

[7] G. Chen, Z.-Q. Wen, and C.-W. Qiu. Superoscillation: from physics to optical applications. Light: Science \& Applications, 8(56):5272, 2019.

[8] N. Franch, J. Canals, V. Moro, A. Villá, A. Romano-Rodríguez, J. D. Prades, J. Gülink, D. Bezdhlyakh, A. Waag, K. Kluczyk-Korch, M. Auf der Maur, A. Di Carlo, and Á. Diéguez. Nano-Illumination Microscopy: a technique based on scanning with an array of individually addressable nanoLEDs. Optics Express, 28(13):19044 - 19057, 2020.

[9] M. Mikulics and H. Hardtdegen. Nano-LED array fabrication suitable for future single photon lithography. Nanotechnology, 26:185302, 2015.

[10] M. Mikulics, Y.C. Arango, A. Winden, R. Adam, A. Hardtdegen, D. Grützmacher, E. Plinski, D. Gregušová, J. Novák, P. Kordoš, A. Moonshiram, M. Marso, Z. Sofer, H. Lüth, and H. Hardtdegen. Direct electro-optical pumping for hybrid CdSe nanocrystal/III-nitride based nano-lightemitting diodes. Applied Physics Letters, 108:061107, 2016.

[11] M. Mikulics, A. Winden, M. Marso, A. Moonshiram, H. Lüth, D. Grützmacher, and H. Hardtdegen. Nano-light-emitting-diodes based on InGaN mesoscopic structures for energy saving optoelectronics. Applied Physics Letters, 109:041103, 2016.

[12] S. Mariana, J. Gülink, G. Handmana, F. Yu, K. Strempel, H. Spende, N. Yulianto, T. Granz, J.D. Prades, E. Peiner, H.S. Wasisto, and A. Waag. Vertical GaN Nanowires and Nanoscale LightEmitting-Diode Arrays for Lighting and Sensing Applications. ACS Applied Nano Materials, 2:4133-4142, 2019.

[13] W. C. Chong, W. K. Cho, Z. J. Liu, C. H. Wang, and K. M. Lau. 1700 Pixels Per Inch (PPI) Passive-Matrix Micro-LED Display Powered by ASIC. In 2014 IEEE Compound Semiconductor Integrated Circuit Symposium (CSICS), pages 1-4, 2014.

[14] J. Day, J. Li, D.Y.C. Lie, C. Bradford, J.Y. Lin, and H.X. Jiang. III-Nitride full-scale highresolution microdisplays. Applied Physics Letters, 99(3):031116, 2011.

[15] J. Bai, Y. Cai, P. Feng, P. Fletcher, X. Zhao, and T. Zhu, C.and Wang. A Direct Epitaxial Approach To Achieving Ultrasmall and Ultrabright InGaN Micro Light-Emitting Diodes ( $\mu$ LEDs). ACS Photonics, 7(2):411 - 415, 2020.

[16] J.F. C. Carreira, 1, A. D. Griffiths, E. Xie, B. J. E. Guilhabert, J. Herrnsdorf, R. K. Henderson, E. E. Gu, M. J. Strain, and M. D. Dawson. Direct integration of micro-LEDs and a SPAD 
detector on a silicon CMOS chip for data communications and time-of-flight ranging. Optics Express, 28(5):6909, 2020.

[17] J. J. D. McKendry, D. Massoubre, S. Zhang, B. R. Rae, R. P. Green, E. Gu, R. K. Henderson, A. E. Kelly, and M. D. Dawson. Visible-Light Communications Using a CMOS-Controlled Micro-LightEmitting-Diode Array. Journal of Lightwave Technology, 30(1):61 - 67, 2012.

[18] Q. Jiao, Z. Chen, Y. Feng, S. Li, S. Jiang, J. Li, Y. Chen, T. Yu, X. Kang, B. Shen, and G. Zhang. The effects of nanocavity and photonic crystal in InGaN/GaN nanorod LED arrays. Nanoscale Research Letters, 11:340, 2016.

[19] S. Fox, S. O'Kane, S. Lis, and D. Allsopp. Designing ingan/gan nano-led arrays for étendue-limited applications. Physica Status Solidi C, 12(4-5):456 - 459, 2015.

[20] J. Kupec, R. L. Stoop, and B. Witzigmann. Light absorption and emission in nanowire array solar cells. Optics Express, 18(26):27591, 2010.

[21] M.-L. Kuo, Y.-S. Kim, M.-L. Hsieh, and S.-Y. Lin. Efficient and Directional Nano-LED Emission by a Complete Elimination of Transverse-Electric Guided Modes. Nanoletters, 11(2):476 - 481, 2011.

[22] Dassault Systèmes . CST Studio Suite.

[23] J. Gülink, S. Bornemann, H. Spende, M. Auf der Maur, A. Di Carlo, J. D. Prades, H. S. Wasisto, and A. Waag. InGaN/GaN nanoLED Arrays as a Novel Illumination Source for Biomedical Imaging and Sensing Applications. Proceedings, 2(13):892, 2018.

[24] M. R. Querry. Optical Constants, 1985.

[25] T. Kawashima, H. Yoshikawa, and s. Adachi. Optical properties of hexagonal GaN. Journal of Applied Physics, 82:3528 - 3335, 1997.

[26] T. A. F. König, P. A. Ledin, J. Kerszulis, M. A. Mahmoud, M. A. El-Sayed, J. R. Reynolds, and V. V. Tsukruk. Electrically Tunable Plasmonic Behavior of Nanocube-Polymer Nanomaterials Induced by a RedoxActive Electrochromic Polymer. ACS Nano, 8(6):6182 - 6192, 2014.

[27] Microchem SU-8 3000 Data Sheet, 2011.

[28] P. Giannios, K.G. Toutouzas, M. Matiatou, K. Stasinos, G.C. Konstadoulakis, M.M.and Zografos, and K. Moutzouris. Visible to near-infrared refractive properties of freshly-excised human-liver tissues: marking hepatic malignancies. Scientific Reports, 6:27910, 2016.

[29] S. Jahani and J. Zubin. Photonic skin-depth engineering. Journal of the Optical Society of America $B, 32(7): 1346-1353,2015$. 